\title{
Time Evolution of Folded (2+1)-Dimensional Solitary Waves
}

\author{
Song-Hua Ma, Yi-Pin Lu, Jian-Ping Fang, and Zhi-Jie Lv \\ Department of Physics, Zhejiang Lishui University, Lishui 323000, China \\ Reprint requests to S.-H. M.; E-mail: msh6209@yahoo.com.cn
}

Z. Naturforsch. 64a, 309 - 314 (2009); received March 31, 2008 / revised September 29, 2008

\begin{abstract}
With an extended mapping approach and a linear variable separation approach, a series of solutions (including the Weierstrass elliptic function solutions, solitary wave solutions, periodic wave solutions and rational function solutions) of the (2+1)-dimensional modified dispersive water-wave system (MDWW) is derived. Based on the derived solutions and using some multi-valued functions, we find a few new folded solitary wave excitations.
\end{abstract}

Key words: Extended Mapping Approach; Variable Separation Approach; Modified Dispersive Water-Wave System; Folded Solitary Wave Excitations.

PACS numbers: 05.45.Yv, 03.65.Ge

\section{Introduction}

Soliton theory is an important aspect of nonlinear science. It is widely applied in many natural sciences such as chemistry, biology, mathematics, communication, and in particular in almost all branches of physics like fluid dynamics, plasma physics, field theory, optics, and condensed matter physics [1-6]. Previously, much efforts have been focused on single valued localized excitations, such as solitoffs, dromions, rings, lumps, breathers, instantons, peakons, compactons, localized chaotic, and fractal patterns [7-20]. However, there are various complicated phenomena in the real natural world like bubbles on a fluid surface or ocean waves, which are folded and obviously cannot be described by single valued functions. In [21], Tang and Lou introduced some multi-valued localized excitations to describe folded solitary waves and to define a new type of soliton - foldon. Actually, the simple foldons in lower dimensions can be equivalently called the loop solitons which can be found in many $(1+1)$ dimensional integrable models $[22,23]$ and have been applied in some physical branches like quantum theory, string theory, and particle physics $[24,25]$. But for these lower-dimensional foldons we know little on foldons in higher dimensions. In this paper, by using some multi-valued functions, we found some new folded localized excitations in the (2+1)-dimensional modified dispersive water-wave system (MDWW)

$$
u_{t y}+u_{x x y}-2 v_{x x}-\left(u^{2}\right)_{x y}=0,
$$

$$
v_{t}-v_{x x}-2(u v)_{x}=0 .
$$

The MDWW system is used to model nonlinear and dispersive long gravity waves travelling in two horizontal directions in shallow water with uniform depth. It can also be derived from the celebrated Kadomtsev-Petviashvili (KP) equation by a symmetry constraint [26].

\section{New Exact Solutions of the (2+1)-Dimensional MDWW System}

In this section, we give some exact solutions of the MDWW system, including solitary wave solutions, trigonometric function solutions, rational solutions, and Weierstrass function solutions.

Letting $f \equiv f(\xi(x))$ and $g \equiv g(\xi(x))$, where $\xi \equiv$ $\xi(x)$ is a undetermined function for the independent variables $x \equiv\left(x_{0}=t, x_{1}, x_{2}, \cdots, x_{m}\right)$, the projective Riccati equation $[27,28]$ is defined by

$$
f^{\prime}=p f g, \quad g^{\prime}=q+p g^{2}-r f
$$

where $p^{2}=1$ and $q$ and $r$ are two real constants. When $p=-1$ and $q=1$, (2) reduces the coupled equations given in [27] and the following relation between $f$ and $g$ holds if $\delta= \pm 1$ and $q \neq 0$ :

$$
g^{2}=-\frac{1}{p}\left[q-2 r f+\frac{r^{2}+\delta}{q} f^{2}\right] .
$$

Equation (3) had been discussed in [28]. In this paper, we discuss other cases. 
Lemma. If the condition of (3) holds with other choices of $\delta$, the projective Riccati equation (2) has the following solutions:

Case 1: If $\delta=-r^{2}$, the Weierstrass elliptic function solution

$$
f=\frac{q}{6 r}+\frac{2}{p r} \wp(\xi), \quad g=\frac{12 \wp^{\prime}(\xi)}{q+12 p \wp(\xi)}
$$

is admitted. Here $p= \pm 1$, the Weierstrass elliptic function $\wp(\xi)=\wp\left(\xi ; g_{2}, g_{3}\right)$ satisfies $\wp^{2}(\xi)=4 \wp^{3}(\xi)-$ $g_{2} \wp(\xi)-g_{3}$, and $g_{2}=\frac{g^{2}}{12}, g_{3}=\frac{p q^{3}}{216}$.

Case 2: If $\delta=-\frac{r^{2}}{25}$, the projective Riccati equation (2) has the Weierstrass elliptic function solution

$$
f=\frac{5 q}{6 r}+\frac{5 p q^{2}}{72 r \wp(\xi)}, g=-\frac{q \wp^{\prime}(\xi)}{\wp(\xi)(p q+12 \wp(\xi))},
$$

where $p= \pm 1$. Both $q$ and $r$ in (4) and (5) are arbitrary constants.

Case 3: If $\delta=h^{2}-s^{2}$ and $p q<0$, (2) has the solitary wave solution

$$
\begin{aligned}
& f=\frac{q}{r+s \cosh (\sqrt{-p q} \xi)+h \sinh (\sqrt{-p q} \xi)}, \\
& g=-\frac{\sqrt{-p q}}{p} \frac{s \sinh (\sqrt{-p q} \xi)+h \cosh (\sqrt{-p q} \xi)}{r+s \cosh (\sqrt{-p q} \xi)+h \sinh (\sqrt{-p q} \xi)},
\end{aligned}
$$

where $p= \pm 1, r, s$, and $h$ are arbitrary constants.

Case 4: If $\delta=-h^{2}-s^{2}$ and $p q>0$, (2) has the trigonometric function solution

$$
\begin{aligned}
& f=\frac{q}{r+s \cos (\sqrt{p q} \xi)+h \sin (\sqrt{p q} \xi)}, \\
& g=\frac{\sqrt{p q}}{p} \frac{s \sin (\sqrt{p q} \xi)-h \cos (\sqrt{p q} \xi)}{r+s \cos (\sqrt{p q} \xi)+h \sin (\sqrt{p q} \xi)},
\end{aligned}
$$

where $p= \pm 1, r, s$, and $h$ are arbitrary constants.

Case 5: If $q=0$, (2) has the rational solution

$$
\begin{aligned}
& f=\frac{2}{p r \xi^{2}+C_{1} \xi-C_{2}}, \\
& g=-\frac{2 p r \xi+C_{1}}{\left(p r \xi^{2}+C_{1} \xi-C_{2}\right) p},
\end{aligned}
$$

where $p= \pm 1, r, C_{1}$, and $C_{2}$ are arbitrary constants.

We now introduce the mapping approach via the above projective Riccati equation. The basic ideal of the algorithm is as follows. For a given nonlinear partial differential equation (NPDE) with the independent variables $x=\left(x_{0}=t, x_{1}, x_{2}, \cdots, x_{m}\right)$, and the dependent variable $u$, has the form

$$
P\left(u, u_{t}, u_{x_{i}}, u_{x_{i} x_{j}}, \cdots\right)=0,
$$

where $P$ is in general a polynomial function of its arguments, and the subscripts denote the partial derivatives. We assume that its solution is written in terms of the standard truncated Painlevé expansion, namely

$$
\begin{aligned}
u= & A_{0}(x) \\
& +\sum_{i=1}^{n}\left[A_{i}(x) f(\xi(x))+B_{i}(x) g(\xi(x))\right] f^{i-1}(\xi(x)) .
\end{aligned}
$$

Here $A_{0}(x), A_{i}(x), B_{i}(x)(i=1, \cdots, n)$ are functions of $x$, and $f, g$ satisfy the projective Riccati equation (2).

To determine $u$ explicitly, one proceeds as follow: First similar to the usual mapping approach, we can determine $n$ by balancing the highest-order partial differential terms with the highest nonlinear terms in (9). Second, substituting (10) together with (2) and (3) into the given NPDE, collecting the coefficients of polynomials of $f^{i} g^{i}$ and eliminating each of them, we can derive a set of partial differential equations for $A_{0}(x)$, $A_{i}(x), B_{i}(x)(i=1, \cdots, n)$ and $\xi(x)$. Third, to calculate $A_{0}(x), A_{i}(x), B_{i}(x)(i=1, \cdots, n)$ and $\xi(x)$, we solve these partial differential equations. Finally, substituting $A_{0}(x), A_{i}(x), B_{i}(x)(i=1, \cdots, n), \xi(x)$ and the solutions (4) - (8) into (10), one obtains solutions of the given NPDE.

First, let us make a transformation of (1): $v=u_{y}$. Substituting this transformation into (1), yields

$$
u_{y t}-u_{x x y}-\left(u^{2}\right)_{x y}=0 .
$$

Now we apply the mapping approach to (11). By the balancing procedure, ansatz (10) becomes

$$
u=F+G f(\xi(x, y, t))+H g(\xi(x, y, t)),
$$

where $F, G, H$, and $\xi$ are arbitrary functions of $(x, y, t)$ to be determined. Substituting (12) together with (2) and (3) into (11), collecting the coefficients of the polynomials of $f^{i} g^{i}(i=0,1,2 \cdots, j=0,1,2 \cdots)$ and setting each of the coefficients equal to zero, we can derive a set of partial differential equations for $F, G$, $H$, and $\xi$. It is difficult to obtain the general solutions of these algebraic equations based on the solutions of (2). Fortunately, in the special case if setting $\xi=\chi(x, t)+\varphi(y)$, where $\chi \equiv \chi(x, t), \varphi \equiv \varphi(y)$ 
are two arbitrary variable separated functions of $(x, t)$ and $y$, respectively, we can obtain solutions of (1).

Case 1. For $\delta=-r^{2}$, the Weierstrass elliptic function solutions are

$$
\begin{aligned}
& u_{1}=\frac{1}{2} \frac{\chi_{t}-\chi_{x x}}{\chi_{x}}-\frac{1}{2} p \chi_{x} g(\xi), \\
& v_{1}=\frac{1}{2} \operatorname{pr}_{x} \varphi_{y} f(\xi)
\end{aligned}
$$

where $p= \pm 1, f, g$ are expressed by (4).
Case 2. For $\delta=-\frac{r^{2}}{25}$, another set of Weierstrass elliptic function solutions are found

$$
\begin{gathered}
u_{2}=\frac{1}{2} \frac{\chi_{t}-\chi_{x x}}{\chi_{x}}+\frac{1}{5} \frac{\sqrt{-6 p q} r \chi_{x} f(\xi)}{q}-\frac{1}{2} p \chi_{x} g(\xi), \\
v_{2}=-\frac{1}{5} \frac{\sqrt{-6 p q} p r \chi_{x} \varphi_{y} f(\xi) g(\xi)}{q} \\
+\frac{6}{25} \frac{p r^{2} \chi_{x} \varphi_{y} f^{2}(\xi)}{q}+\frac{1}{2} \operatorname{pr} \chi_{x} \varphi_{y} f(\xi),
\end{gathered}
$$

where $p= \pm 1, q$ and $r$ are arbitrary constants, $f, g$ are expressed by (5).

Case 3. For $\delta=h^{2}-s^{2}$ and $p q=-1$, the solitary wave solutions are

$$
\begin{aligned}
& u_{3}= \frac{1}{2} \frac{r\left(\chi_{t}-\chi_{x x}\right)+\cosh (\chi+\varphi)\left[s\left(\chi_{t}-\chi_{x x}\right)+h \chi_{x}^{2}\right]+\sinh (\chi+\varphi)\left[h\left(\chi_{t}-\chi_{x x}\right)+s \chi_{x}^{2}\right]}{\chi_{x}(r+s \cosh (\chi+\varphi)+h \sinh (\chi+\varphi))} \\
&+\frac{1}{2} \frac{\chi_{x} \sqrt{r^{2}+h^{2}-s^{2}}}{r+s \cosh (\chi+\varphi)+h \sinh (\chi+\varphi)}, \\
& v_{3}=-\frac{1}{2} \frac{\chi_{x} \varphi_{y}\left[\cosh (\chi+\varphi)\left(h \sqrt{r^{2}+h^{2}-s^{2}}-s r\right)\right]}{[r+s \cosh (\chi+\varphi)+h \sinh (\chi+\varphi)]^{2}}-\frac{1}{2} \frac{\chi_{x} \varphi_{y}\left[\sinh (\chi+\varphi)\left(s \sqrt{r^{2}+h^{2}-s^{2}}-h r\right)-s^{2}+h^{2}\right]}{[r+s \cosh (\chi+\varphi)+h \sinh (\chi+\varphi)]^{2}}
\end{aligned}
$$

with two arbitrary functions $\chi(x, t)$ and $\varphi(y)$, while $r, s, h$ are arbitrary constants.

Case 4. For $\delta=-h^{2}-s^{2}$ and $p q=1$, the trigonometric function solutions are

$$
\begin{gathered}
u_{4}=\frac{1}{2} \frac{\chi_{t}-\chi_{x x}}{\chi_{x}}+\frac{1}{2} \frac{\chi_{x}\left[\sqrt{s^{2}+h^{2}-r^{2}}+h \cos (\chi+\varphi)-s \sin (\chi+\varphi)\right]}{r+s \cos (\chi+\varphi)+h \sin (\chi+\varphi)}, \\
v_{4}=-\frac{1}{2} \frac{\chi_{x} \varphi_{y}\left[\cos (\chi+\varphi)\left(h \sqrt{-r^{2}+h^{2}+s^{2}}+s r\right)\right]}{[r+s \cos (\chi+\varphi)+h \sin (\chi+\varphi)]^{2}}-\frac{1}{2} \frac{\chi_{x} \varphi_{y}\left[\sin (\chi+\varphi)\left(-s \sqrt{-r^{2}+h^{2}+s^{2}}+h r\right)+s^{2}+h^{2}\right]}{[r+s \cos (\chi+\varphi)+h \sin (\chi+\varphi)]^{2}}
\end{gathered}
$$

with two arbitrary functions $\chi(x, t)$ and $\varphi(y)$, while $r, s, h$ are arbitrary constants.

Case 5. For $q=0$, the rational solutions are

$$
\begin{aligned}
u_{5}= & \frac{1}{2} \frac{\chi_{t}-\chi_{x x}}{\chi_{x}}+\frac{1}{2} \frac{\chi_{x}\left[p \sqrt{C_{1}^{2}+4 C_{2} p r}+2 p r(\chi+\varphi)+C_{1}\right]}{p r(\chi+\varphi)^{2}+C_{1}(\chi+\varphi)-C_{2}}, \\
v_{5}= & -\frac{1}{2} \frac{\chi_{x} \varphi_{y}\left[\sqrt{C_{1}^{2}+4 C_{2} p r}\left(2 p^{2} r \chi+2 p^{2} r \varphi+p C_{1}\right)\right]}{\left[p r(\chi+\varphi)^{2}+C_{1}(\chi+\varphi)-C_{2}\right]^{2}} \\
& -\frac{1}{2} \frac{\chi_{x} \varphi_{y}\left[2 p^{2} r^{2}(\chi+\varphi)^{2}+2 p r C_{1}(\chi+\varphi)+2 C_{2} p r+C_{1}^{2}\right]}{\left[p r(\chi+\varphi)^{2}+C_{1}(\chi+\varphi)-C_{2}\right]^{2}}
\end{aligned}
$$

with two arbitrary functions $\chi(x, t)$ and $\varphi(y)$, while $p= \pm 1, C_{1}, C_{2}$, and $r$ are arbitrary constants. 


\section{Some Novel Folded Localized Excitations in the (2+1)-Dimensional MDWW System}

Due to the arbitrariness of the functions $\chi(x, t), \varphi(y)$ included in the above cases, the physical quantities $u$ and $v$ may possess rich structures. In this section, we mainly discuss some folded localized coherent excitations in the (2+1)-dimensional MDWW system. For simplicity, we only discuss the field $v_{5}$ of (22), namely

$$
\begin{aligned}
V=v_{5}= & -\frac{1}{2} \frac{\chi_{x} \varphi_{y}\left[\sqrt{C_{1}^{2}+4 C_{2} p r}\left(2 p^{2} r \chi+2 p^{2} r \varphi+p C_{1}\right)\right]}{\left[p r(\chi+\varphi)^{2}+C_{1}(\chi+\varphi)-C_{2}\right]^{2}} \\
& -\frac{1}{2} \frac{\chi_{x} \varphi_{y}\left[2 p^{2} r^{2}(\chi+\varphi)^{2}+2 p r C_{1}(\chi+\varphi)+2 C_{2} p r+C_{1}^{2}\right]}{\left[p r(\chi+\varphi)^{2}+C_{1}(\chi+\varphi)-C_{2}\right]^{2}} .
\end{aligned}
$$

\subsection{Folded Excitations}

In order to construct kinds of interesting folded localized excitations, we introduce some suitable multivalued functions [21]. For instance,

$$
\chi_{x}=\sum_{j=1}^{M} V_{j}\left(\varepsilon-c_{j} t\right), \quad x=\varepsilon+\sum_{j=1}^{M} P_{j}\left(\varepsilon-c_{j} t\right)
$$

where $c_{j}(j=1,2, \cdots, M)$ are arbitrary constants, $V_{j}$ and $P_{j}$ are localized excitations with the properties $V_{j}( \pm \infty)=0, P_{j}( \pm \infty)=$ const. we also treat the func-
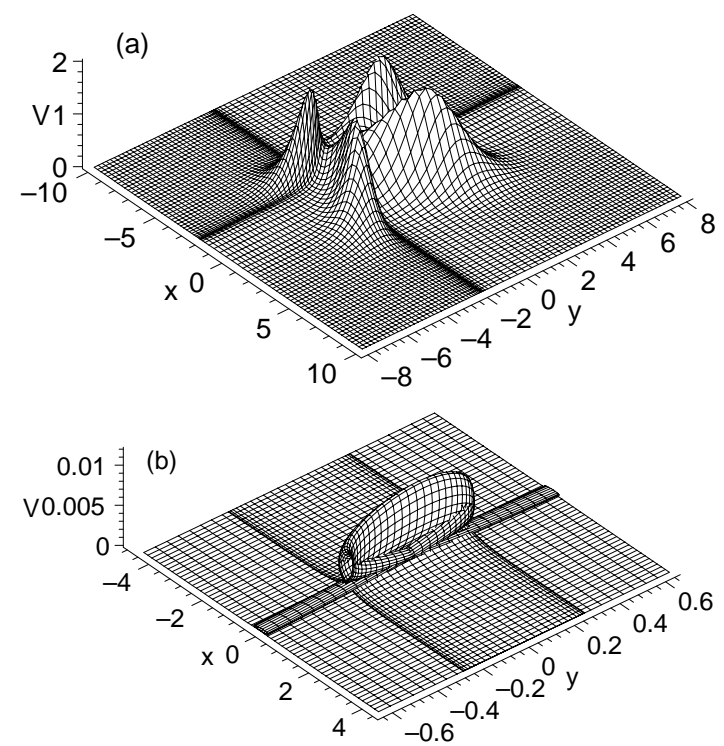

Fig. 1. Two types of folded localized excitation are depicted for the field $V$ from (23) at time $t=0$ with the choices: (a) $\chi_{x}=-\operatorname{sech}^{2}(\varepsilon-\mathrm{ct}), x=\varepsilon-\operatorname{sech}(\varepsilon-c t), \varphi_{y}=\operatorname{sech}^{2}(\zeta)$, $y=\zeta-0.8 \operatorname{sech}(\zeta)$; we set $p=1, r=1, C_{1}=2, C_{2}=1$; (b) same $\chi_{x}$ and $\varphi_{y}$ with (a), however, $x=\varepsilon-0.8 \tanh (\varepsilon-$ ct), $y=\varepsilon-0.8 \tanh (\zeta)$, and using $p=1, r=1, C_{1}=0$, $C_{2}=1$. tion $\varphi(y)$ in this way,

$$
\varphi_{y}=\sum_{j=1}^{N} U_{j}(\zeta), \quad y=\zeta+\sum_{j=1}^{N} Q_{j}(\zeta)
$$

In Fig. 1, two types of folded solitary waves are presented for the field $V$ determined by (23). The related functions chosen in the plots are directly given in the figure captions.

\subsection{The Evolution of Folded Solitary Waves}

Now we focus our attention on the intriguing evolution of two folded solitary waves for the solution $V$. If we choose $\chi$ and $\varphi$ to be some appropriate multivalued functions, then we find that the interactions among the folded solitary waves are completely elastic. For instance, if we choose $\chi$ and $\varphi$ as

$$
\begin{aligned}
& \chi_{x}=-\operatorname{sech}(\varepsilon-t)^{2}-0.7 \operatorname{sech}(\varepsilon+t)^{2}, \\
& x=\varepsilon-\operatorname{sech}(\varepsilon-t)-0.7 \operatorname{sech}(\varepsilon+t), \\
& \varphi_{y}=\operatorname{sech}(\zeta)^{2}, \quad y=\zeta-0.7 \operatorname{sech}(\zeta),
\end{aligned}
$$

we can derive the time evolution of the folded solitary waves for the physical quantity $V$ as presented in Fig. 2 with fixed parameters $p=1, r=1, C_{1}=2, C_{2}=1$, at different times: (a) $t=-13$; (b) $t=-8$; (c) $t=0$; (d) $t=8$; (e) $t=13$.

Furthermore, if we choose $\chi$ and $\varphi$ as

$$
\begin{aligned}
& \chi_{x}=-\operatorname{sech}(\varepsilon-t)^{2}-0.6 \operatorname{sech}(\varepsilon+t)^{2}, \\
& x=\varepsilon-\tanh (\varepsilon-t)-0.6 \tanh (\varepsilon+t), \\
& \varphi_{y}=\operatorname{sech}(\zeta)^{2}, \quad y=\zeta-0.6 \tanh (\zeta),
\end{aligned}
$$

we can derive another time evolution of folded solitary waves for the physical quantity $V$ presented in Fig. 3 with fixed parameters $p=1, r=1, C_{1}=0, C_{2}=1$, at 

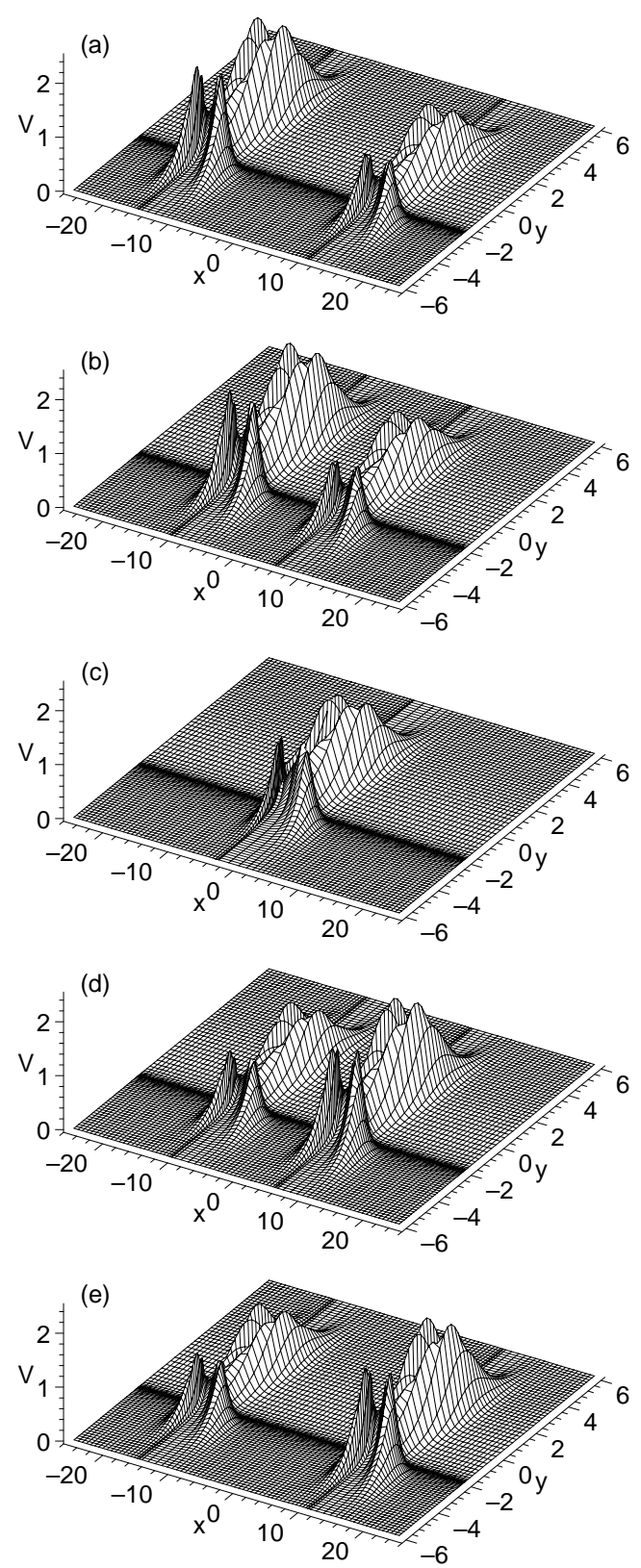

Fig. 2. Evolutional profile of two folded solitary waves for the solution $V$ defined by (23) with condition (26) at different times: (a) $t=-13$; (b) $t=-8$; (c) $t=0$; (d) $t=8$; (e) $t=13$.

different times: (a) $t=-3$; (b) $t=-2.3$; (c) $t=-0.8$; (d) $t=0$; (e) $t=2.3$; (f) $t=3$. From Fig. 2 and Fig. 3 , one finds that the interactions of the two folded solitary waves are completely elastic since their amplitudes, velocities, and wave shapes do not undergo any change after their collision.

\section{Summary and Discussion}

In summary, via the extended mapping approach and a linear variable separation approach, we find some new exact solutions of the (2+1)-dimensional modified dispersive water-wave system. Abundant localized coherent soliton structures of the solution $V$ of (23) like dromions, peakons, breathers, instantons, etc., can be easily constructed by choosing suitable functions. Except for the single-valued localized excitation we find a new type of multi-valued localized excitation, i. e., folded solitary waves and/or foldon excitations for the (2+1)-dimensional modified dispersive water-wave system. To our knowledge, the folded solitary waves and/or foldon excitations for the (2+1)-dimensional modified dispersive water-wave system have not been reported in the previous literature. The time evolution of the two folded solitary waves displayed in Fig. 2 is different from the ones presented in the previous work.

\section{Acknowledgements}

The authors would like to thank Professor Jie-Fang Zhang for his fruitful and helpful suggestions. This work has been supported by the Natural Science Foundation of Zhejiang Province (Grant Nos. Y604106, Y606128), the Scientific Research Fund of Zhejiang Provincial Education Department of China (Grant No. 20070568), the undergraduate innovation project of science and technology of Zhejiang Province, the project of seeding grants of Zhejiang Province, and Natural Science Foundation of Zhejiang Lishui University (Grant No. KZ08001). 

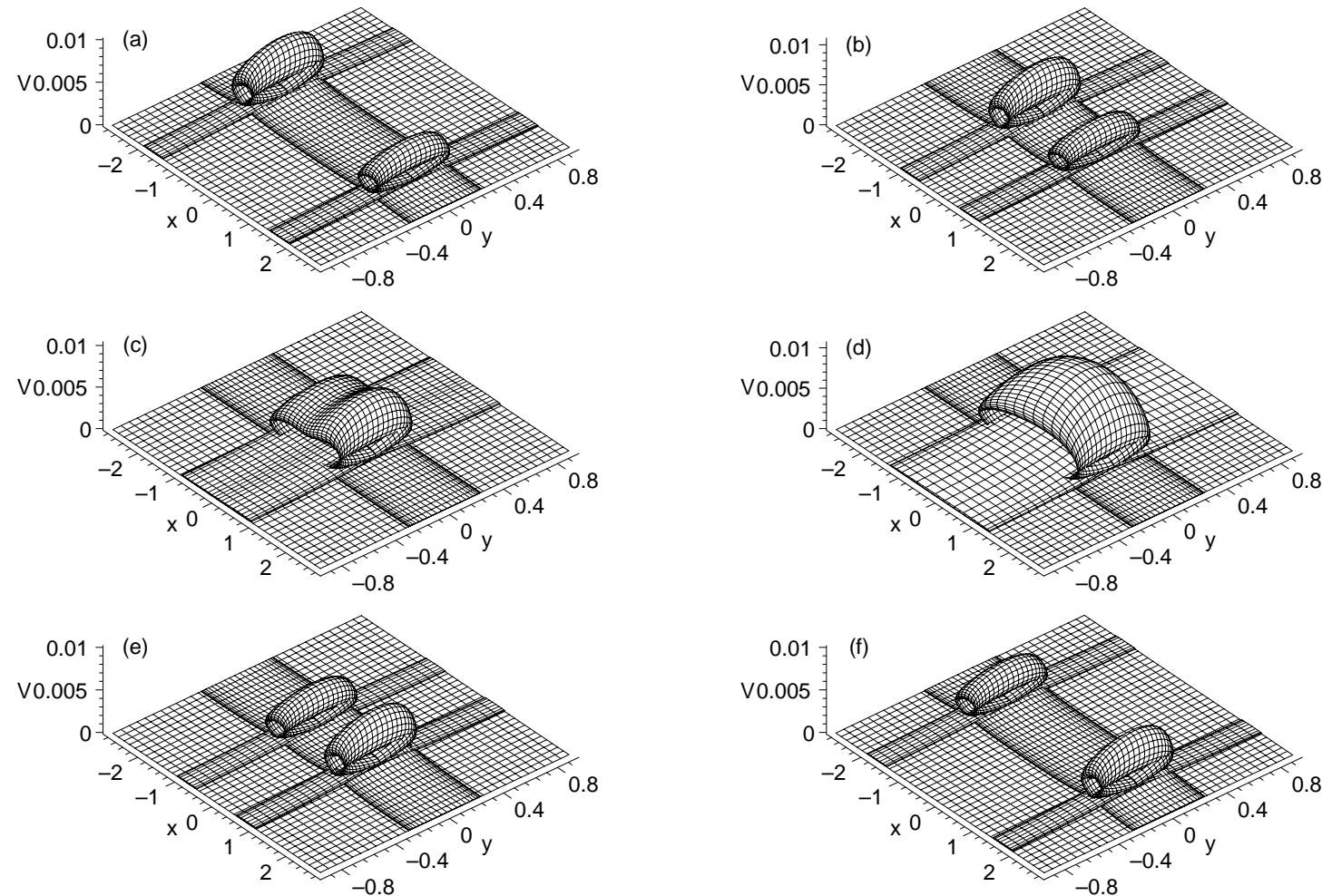

Fig. 3. Another evolutional profile of the two folded solitary waves for the solution $V$ defined by (23) with condition (27) at different times: (a) $t=-3$; (b) $t=-2.3$; (c) $t=-0.8$; (d) $t=0$; (e) $t=2.3$; (f) $t=3$.

[1] S. Y. Lou and X. Y. Tang, Chin. Phys. Lett. 19, 769 (2002).

[2] I. Loutsenko and D. Roubtsov, Phys. Rev. Lett. 78, 3001 (1997).

[3] M. Tajiri and H. Maesono, Phys. Rev. E 55, 3351 (1997).

[4] D. W. C. Lai and K. W. Chow, J. Phys. Soc. Jpn. 70, 666 (2001)

[5] V. N. Serkin, Opt. Commun. 192, 237 (2001).

[6] V.E. Zakharov and J. Li, Appl. Mech. Tech. Phys. 9, 190 (1998).

[7] Inverse Probl. 3, 371 (1987).

[8] S. Wang, X. Y. Tang, and S. Y. Lou, Chaos, Solitons, and Fractals 19, 769 (2004)

[9] C. L. Zheng, Commun. Theor. Phys. 40, 25 (2003).

[10] C. L. Zheng and J. M. Zhu, Commun. Theor. Phys. 39, 261 (2003).

[11] C. L. Zheng and J. F. Zhang, Commun. Theor. Phys. 40, 385 (2003).

[12] J.P. Fang and C.L. Zheng, Z. Naturforsch. 60a, 245 (2005).

[13] J. P. Fang and C. L. Zheng, Acta Phys. Sin. 54, 2991 (2005).

[14] J. P. Fang and C. L. Zheng, Z. Naturforsch. 60a, 250 (2005).
[15] S. H. Ma, X. H. Wu, J. P. Fang, and C. L. Zheng, Z. Naturforsch. 61a, 249 (2006).

[16] S. H. Ma and J. P. Fang, Acta. Phys. Sin. 55, 37 (2006).

[17] S. H. Ma, J. P. Fang, and C. L. Zheng, Z. Naturforsch. 62a, 8 (2007).

[18] S. H. Ma, J. Y. Qiang, and J. P. Fang, Acta. Phys. Sin. 56, 0620 (2007).

[19] S. H. Ma, J. P. Fang, and H. P. Zhu, Acta. Phys. Sin. 56, 4319 (2007).

[20] S. H. Ma, J. Y. Qiang, and J. P. Fang, Commun. Theor. Phys. 48, 662 (2007).

[21] X. Y. Tang and S. Y. Lou, Commun. Theor. Phys. 40, $62(2003)$.

[22] V. O. Vakhnenkl, J. Phys. A. Math. Gen. 25, 4181 (1992).

[23] V. O. Vakhnenkl and E. J. Parkes, Nonlinearity 11, 1457 (1998).

[24] S. Matsutani, Mod. Phys. Lett. A 10, 717 (1995).

[25] M. Schleif and R. Wunsch, Eur. Phys. J. A 1, 171 (1998).

[26] V. G. Durovsky and E. G. Konopelchenko, J. Phys. A 27, 4619 (1994).

[27] R. Conte and M. Musette, J. Phys. A Math. Gen. 25, 5609 (1992).

[28] Z. T. Fu, S. D. Liu, and S. K. Liu, Chaos, Solitons, and Fractals 20, 301 (2004). 